\title{
CHARACTERISTIC APPROXIMATION PROPERTIES OF QUADRATIC IRRATIONALS
}

\section{W. B. JURKAT AND A. PEYERIMHOFF}

Universitat ULM (MNH)

Abt. f. Mathematic I

$7900 \mathrm{U} 1 \mathrm{~m}$ (Donau)

Oberer Eselsberg, Germany

(Received April 19, 1978)

ABSTRACT. Some characteristic approximation properties of quadratic irrationals are studied in this paper. It is shown that the limit points of the sequence $\delta_{n}$ form a subset $C(x)$, and $D(x)$ can be generated from $C(x)$ in a relatively simple way. Another proof of Lekkerkerker's theorem is given using relations between $\delta_{n-1}, \delta_{n}, \delta_{n+1}$ which are independent of $\mathrm{x}$ and $\mathrm{n}$.

KEY WORDS AND PHRASES. Quadratic Irrationals, Approximation of numbers, Badly Approximable Numbers.

AMS (MOS) SUBJECT CLASSIFICATION (1970) CODES. 10F05, $10 F 35$. 
0 . Throughout this paper $\mathrm{x}$ will denote a real irrational number. We introduce

$$
|| x||=\min _{k \in \mathbb{Z}}|x-k| \quad, \quad r(x)=x-\left[x+\frac{1}{2}\right]
$$

which implies $\quad r(x) \epsilon\left[-\frac{1}{2}, \frac{1}{2}\right), \quad|r(x)|=|| x||$.

Given $\mathrm{x}$, the sequence $\mathrm{n}|| \mathrm{nx}||, n \in \mathbf{N}$, contains bounded subsequences (e.g. $\mathrm{n}|| \mathrm{nx}||<1 / \sqrt{5}$ for infinitely many $\mathrm{n}$ by Hurwitz's theorem), and it seems natural to investigate the set $D(x)$ of all its limit points which describes the various qualities of approximation of $x$ by rationals which occur again and again 1 ) A number $x$ is "well approximable" if $0 \in \mathrm{D}(\mathrm{x})$ (e.g. if $\mathrm{x}=\mathrm{e}=2.71 \ldots$ or if $\mathrm{x}$ is a Liouville number) and "badly approximable" if $0 \notin D(x)$. If $0 \in D(x)$ then 2$)$ $D(x)=[0, \infty)$, hence interesting numbers in this context are the badly approximable numbers.

Let $x$ be represented by the continued fraction $\left[b_{0}, b_{1}, \ldots\right]$, let $A_{n} / B_{n}$ denote its convergents and let

$$
\delta_{n}=\delta_{n}(x)=B_{n}\left|B_{n} x-A_{n}\right|, n \geq-2 \quad\left(\delta_{n}=B_{n}|| B_{n} x|| \text { for } n \geq 1\right)
$$

The limit points of the sequence $\delta_{n}$ form a subset $C(x)$ (which is in a sense constructive) and we shall show that $D(x)$ can be generated from $C(x)$ in a relatively simple way (Theorem 1), so the structure of $C(x)$ is basic in our context.

A theorem of Lekkerkerker [5] shows that for a badly approximable number $x$ the set $C(x)$ is finite if and only if $x$ is a quadratic irrational, and the connection between $C(x)$ and $D(x)$ shows that $D(x)$ is discrete if and only if

1) For results on $\operatorname{infD}(x)$, which is the inverse of Perron's modular function $[5]$, see [1] and the bibiliography of this paper.

2) Let $n_{i}=n_{i}|| n_{i} x||+0$, choose $0<\alpha \in \mathbf{R}$, and let $n_{i}{ }^{*}=n_{i}\left[\sqrt{\frac{\alpha}{n_{i}}}\right]$ Then $\eta_{i}\left[\sqrt{\frac{\alpha}{n_{i}}}\right]^{2}=n_{i}^{*}|| n_{i}^{*} x||$ for $i$ large and $n_{i}\left[\sqrt{\frac{\alpha}{n_{i}}}\right]^{2} \rightarrow \alpha$. Hence $\alpha \in D(x)$. 
$\mathbf{x}$ is (badly approximable and) a quadratic irrational. We will also give another proof of Lekkerkerker's theorem using relations between $\delta_{n-1}, \delta_{n}, \delta_{n+1}$ which are independent of $x$ and $n$ and seem to tell the whole structure of the $\delta_{n}$ 's (Lemma 3, Theorem 3).

\section{THE BASIC FORMULAS.}

Writing $x=\left[b_{0}, b_{1}, \ldots\right]=\left[b_{0}, b_{1}, \ldots, b_{n-1}+\frac{1}{\xi_{n}}\right], \xi_{n}=\left[b_{n}, b_{n+1}, \ldots\right]$ and $\rho_{n}=\frac{B_{n}}{B_{n-1}}, n \geq 1,1 / \rho_{0}=0$ we have for $n \geq 0$ the following well known formulas

$$
\begin{gathered}
\xi_{n}=b_{n}+\frac{1}{\xi_{n+1}} \\
B_{n}\left(B_{n} x-A_{n}\right)=\frac{(-1)^{n}}{\xi_{n+1}+\frac{1}{\rho_{n}}} \\
b_{n+1}=\rho_{n+1}-\frac{1}{\rho_{n}}
\end{gathered}
$$

(cf. [7], 13; (4) is a consequence of $B_{n+1}=b_{n+1} B_{n}+B_{n-1}, n \geq-1$ ).

LEMMA 1. For $\mathrm{n} \geq 1$

$$
\begin{aligned}
& \left.\delta_{n}+\delta_{n-1}<\quad \text { unless } 3\right) \quad n=1, b_{1}=1, \\
& \rho_{n}=\frac{1+\sqrt{1-4 \delta_{n} \delta_{n-1}}}{2 \delta_{n-1}}, \frac{1}{\rho_{n}}=\frac{1-\sqrt{1-4 \delta_{n} \delta_{n-1}}}{2 \delta_{n}} .
\end{aligned}
$$

PROOF. It follows from (2) and (4) that

$$
\xi_{n}+\frac{1}{\rho_{n-1}}=b_{n}+\frac{1}{\xi_{n+1}}+\frac{1}{\rho_{n-1}}=\frac{1}{\xi_{n+1}}+\rho_{n}(n \geq 1) \text {. This and (1) (3) }
$$

3) If $b_{1}=1$ then $\delta_{0}+\delta_{1}=(x-[x])-(x-[x]-1)=1$. 
show that

$$
\delta_{n}+\delta_{n-1}=\frac{\xi_{n+1}+\rho_{n}}{1+\rho_{n} \xi_{n+1}} \quad \text { for } n \geq 1
$$

which implies (5) (note that $\xi_{n+1}>1$ ). In order to prove (6) we note that the foregoing calculations also show that

$$
1-4 \delta_{n} \delta_{n-1}=1-4 \frac{\rho_{n} \xi_{n+1}}{\left(1+\rho_{n} \xi_{n+1}\right)^{2}}=\left(\frac{\rho_{n} \xi_{n+1}-1}{1+\rho_{n} \xi_{n+1}}\right)^{2}
$$

and this leads immediately to (6).

Formulas (4) and (6) suggest the introduction of the function

$$
\phi(\mathrm{x}, \mathrm{y} ; \mathrm{z})=\frac{\sqrt{1-4 \mathrm{xz}}+\sqrt{1-4 \mathrm{yz}}}{2 \mathrm{z}}, \mathrm{z}>0,4 \mathrm{xz}<1,4 \mathrm{yz}<1 .
$$

using this notation, we have

$$
b_{n+1}=\phi\left(\delta_{n-1}, \delta_{n+1} ; \delta_{n}\right), n \geq 0 \quad\left(\delta_{-1}=0\right)
$$

The following properties of $\phi$ will be used in later sections of this paper :

$$
\begin{gathered}
\phi(\mathrm{x}, \mathrm{y} ; \mathrm{z})=\phi(\mathrm{y}, \mathrm{x} ; \mathrm{z}), \\
\phi(\mathrm{x}, \mathrm{y} ; \mathrm{z}) \downarrow \text { (strictly) if } \mathrm{x} \uparrow, \mathrm{y} \uparrow \text { or } \mathrm{z} \uparrow, \\
\phi(\mathrm{x}, 1-\mathrm{z} ; \mathrm{z})=\frac{|2 \mathrm{z}-1|+\sqrt{1-4 \mathrm{xz}}}{2 \mathrm{z},} \\
\phi(\mathrm{x}, 0 ; \mathrm{z})-\phi(\mathrm{x}, 1-\mathrm{z} ; \mathrm{z})=\frac{1-|2 \mathrm{z}-1|}{2 \mathrm{z}}=\left\{\begin{array}{ll}
1 & \text { if } z \leq 1 / 2 \\
\frac{1-\mathrm{z}}{\mathrm{z}}<1 & \text { if } z>1 / 2
\end{array}\right\}
\end{gathered}
$$

In conclusion we mention that (5) contains Vahlen's result (see e.g. [7], §14) 
that at least one of $\delta_{n}, \delta_{n-1}$ is $<1 / 2$, and Borel's result (see [7], §14) that at least one of $\delta_{n-1}, \delta_{n}, \delta_{n+1}$ is $<1 / \sqrt{5}$ follows from (6), (8) and (10). Indeed, if this were not true then one of the $\delta^{\prime}$ 's would be $>1 / \sqrt{5}$ (since $\delta_{n}=\delta_{n+1}=$ $1 / \sqrt{5}$ and (6) would imply $\rho_{n+1}=\frac{\sqrt{5}+1}{2}$, but $\rho_{n}$ is rational) and this and (8) and (10) imply

$$
b_{n+1}=\phi\left(\delta_{n-1}, \delta_{n+1} ; \delta_{n}\right)<\phi(1 / \sqrt{5}, 1 / \sqrt{5} ; 1 / \sqrt{5})=1,
$$

but $\quad b_{n+1} \geq 1$.

\section{THE RELATION BETWEEN $C(x)$ AND $D(x)$.}

In addition to $d(x)$ and $C(x)$ we introduce the sets

$$
\begin{aligned}
& D_{s}(x) \text { : the limit points of the sequence } n r(n x) \text {, } \\
& C_{s}(x) \text { : the limit points of the sequence } B_{n} r\left(B_{n} x\right) \text {. }
\end{aligned}
$$

These sets contain information on the sign of the approximations of $x$ by rationals, and $\mathrm{D}(\mathrm{x})$ or $\mathrm{C}(\mathrm{x})$ is known if $\mathrm{D}_{s}(\mathrm{x})$ or $\mathrm{C}_{\mathrm{s}}(\mathrm{x})$ is known.

Let ||$n x||=|n x-m|$, sign $(n x-m)=\varepsilon$. Then it follows from

$$
\begin{aligned}
& \mathrm{n}=\lambda \mathrm{B}_{\mathrm{k}}+\mu \mathrm{B}_{\mathrm{k}-1} \\
& \mathrm{~m}=\lambda \mathrm{A}_{\mathrm{k}}+\mu \mathrm{A}_{\mathrm{k}-1} \quad, \mathrm{k} \geq-1
\end{aligned}
$$

by Cramer's rule that $\lambda, \mu \in \mathbb{Z}$ and that

$$
\begin{aligned}
& \lambda=\mathrm{n}\left|\mathrm{xB}_{\mathrm{k}-1}-\mathrm{A}_{\mathrm{k}-1}\right|+(-1)^{\mathrm{k}} \varepsilon \mathrm{B}_{\mathrm{k}-1}|| \mathrm{nx}||, \\
& \mu=\mathrm{n}\left|\mathrm{xB}_{\mathrm{k}}-\mathrm{A}_{\mathrm{k}}\right|-(-1)^{\mathrm{k}} \varepsilon \mathrm{B}_{\mathrm{k}}|| \mathrm{nx}|| .
\end{aligned}
$$

THEOREM 1. Let $0 \notin D(x)$. Then $\alpha \in D_{s}(x)$ if and only if 


$$
\alpha=\lambda^{2} \gamma-\lambda \mu \sqrt{1+4 \beta \gamma} \operatorname{sign} \gamma+\mu^{2} \beta,
$$

where $\lambda, \mu \in \mathbb{N}_{0}, \quad(\lambda, \mu) \neq(0,0)$ and $\beta=1$ im $B_{k_{i}-1} r\left(B_{k_{i}-1} x\right)$, $\gamma=\lim B_{k_{i}} r\left(B_{k_{i}} x\right)$ for some sequence $k_{i} \rightarrow \infty$.

COROLLARY. Formula (15) and $B \gamma<0$ show that $\mathrm{D}(\mathrm{x})$ and $\mathrm{C}(\mathrm{x})$ are connected by

$$
\alpha=\left|\lambda^{2}\right| \gamma\left|-\lambda \mu \sqrt{1-4|\beta| \gamma \mid}-\mu^{2}\right| \beta|| .
$$

\section{PROOF of Theorem 1.}

Let $n_{i} r\left(n_{i} x\right)=n_{i}\left(n_{i} x-m_{i}\right) \rightarrow \alpha \in D_{s}(x)$, and select $k_{i} \in N \quad$ (for all large $\left.i\right)$ such that

$$
\begin{aligned}
& B_{k_{i}}|| n_{i} x|| \leq n_{i}|| B_{k_{i}} x||, \\
& B_{k_{i}+1}|| n_{i} x||>n_{i}|| B_{k_{i}+1} x||
\end{aligned}
$$

Define numbers $\lambda_{i}, \mu_{i}$ by (13) (with $n_{i}, m_{i}, k_{i}$ instead of $n, m, k$ ). It follows from (17) and (14) that $\lambda_{i}, \mu_{i} \in \mathbf{N}_{0}$. Condition (17) implies $B_{k_{i}} \leq \mathbf{n}_{i}$ since otherwise || $\mathrm{n}_{i} \mathrm{x}||>|| \mathrm{B}_{\mathrm{k}_{i}} \mathrm{x} \|$ by Lagrange's Theorem ([7], §15) which lead: to a contradiction to (17). On the other hand, it follows from

$$
\begin{aligned}
& || B_{k_{i}+1} x||>\left(B_{k_{i}+1}+B_{k_{i}+2}\right)^{-1}([7], \S 13) \text { and (18) that } \\
& \qquad \frac{n_{i}^{2}}{B_{k_{i}+1}+B_{k_{i}+2}} \leq n_{i}^{2}|| B_{k_{i}+1} x||<B_{k_{i}+1} n_{i}|| n_{i} x||=B_{k_{i}+1}(|\alpha|+o(1))
\end{aligned}
$$

which implies $n_{i} \leq 2|\alpha|^{1 / 2} B_{k_{i}+2}$ for all large $i$.

It follows from $0 \notin D(x)$ and $B_{k}|| B_{k} x||<\frac{1}{b_{k+1}}([7], 13)$ that $b_{k+1}=0(1)$. Hence, there is a constant $C=C(\alpha, x)$ such that 


$$
\mathrm{B}_{\mathrm{k}_{i}} \leq \mathrm{n}_{i} \leq \mathrm{C}(\alpha, \mathrm{x}) \mathrm{B}_{\mathrm{k}_{i}-1} \quad \text { for all large } i
$$

From (19) and (14) we infer that

$$
0 \leq \lambda_{i} \leq K_{1}(\alpha, x) \quad, \quad 0 \leq \mu_{i} \leq K_{2}(\alpha, x)
$$

for constants $\mathrm{K}_{1}, \mathrm{~K}_{2}$ and all large $i$.

By taking subsequences, the foregoing shows that sequences $n_{i} \rightarrow \infty, k_{i} \rightarrow \infty$ exist such that

$$
\left\{\begin{array}{l}
\mathrm{n}_{i} \mathrm{r}\left(\mathrm{n}_{\mathrm{i}} \mathrm{x}\right) \rightarrow \alpha \\
\mathrm{n}_{i}=\lambda \mathrm{B}_{\mathrm{k}_{i}}+\mu \mathrm{B}_{\mathrm{k}_{i}-1}, \mathrm{~m}_{i}=\lambda \mathrm{A}_{\mathrm{k}_{i}}+\mu \mathrm{A}_{\mathrm{k}_{i}-1}, \lambda, \mu \in \mathbb{N}_{0},(\lambda, \mu) \neq(0,0) \\
\mathrm{B}_{\mathrm{k}_{i}-1} r\left(\mathrm{~B}_{\mathrm{k}_{i}-1} \mathrm{x}\right) \rightarrow \beta, \mathrm{B}_{\mathrm{k}_{i}} r\left(\mathrm{~B}_{\mathrm{k}_{i}} \mathrm{x}\right) \rightarrow \gamma .
\end{array}\right.
$$

Let $n_{i}, k_{i}$ satisfy (20). Then (note that $r\left(B_{n} x\right)=B_{n} x-A_{n}$ for $n \geq 1$ )

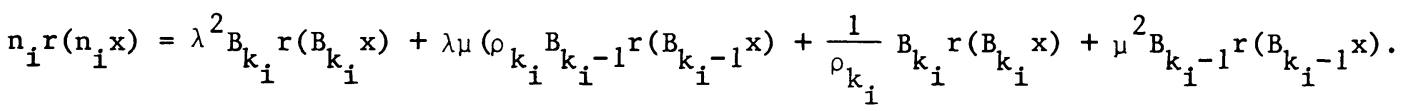
This and (6) show that every $\alpha \in D_{S}$ has a representation (15) and that every number (15) belongs to $\mathrm{D}_{\mathrm{S}}$.

REMARKS. 1. Let $\mathrm{K}>0$. Then the proof of Theorem 1 shows that for every $\alpha \in \mathrm{D}_{\mathrm{S}}(\mathrm{x}),|\alpha| \leq \mathrm{K}$, a representation (15) holds for some $\lambda$ and $\mu$ which are bounded by a constant which depends on $K$ and $x$ only. Hence, if $C(x)$ is discrete (i.e. $C(x)$ is finite since $B_{n}|| B_{n} x|| \leq 1$ ), then $D(x)$ is discrete and vice versa.

2. A slight modification of the proof of Theorem 1 also shows that $\mathrm{n}|| \mathrm{nx}||=\mathrm{n}|\mathrm{nx}-\mathrm{m}|<1 / 2 \quad(\mathrm{n} \in \mathbb{N})$ implies $\mathrm{n} / \mathrm{m}=\mathrm{A}_{v} / \mathrm{B}_{\nu}$ for some $\nu \quad([7], \S 13 ;[2]$ 
Theorem 184; for a more general result compare [4], Proposition 4). In fact, choose $k \geq 1$ such that $B_{k-1}<n \leq B_{k} \quad\left(n=1\right.$ is a trivial case). If $\varepsilon=(-1)^{k}$ and $\mathrm{n}<\mathrm{B}_{\mathrm{k}}$, then (14) leads to the contradiction $0<\lambda<2 \mathrm{n}|| \mathrm{nx}||<1$, hence $\mathrm{n}=\mathrm{B}_{\mathrm{k}}$. If $\varepsilon=(-1)^{\mathrm{k}-1}$, then (14) implies $\mu>0, \lambda>-\mathrm{n}|| \mathrm{nx}||>-1 / 2$, hence $\lambda \geq 0$. But $\lambda<1$ since $\mathrm{n} \leq \mathrm{B}_{\mathrm{k}}$, hence $\mathrm{n}=\mu \mathrm{B}_{\mathrm{k}-1}, \mathrm{~m}=\mu \mathrm{A}_{\mathrm{k}-1}$.

3. THE STRUCTURE OF $\mathrm{C}(\mathrm{x})$ WHEN $\mathrm{x}$ IS A QUADRATIC IRRATIONALITY.

We show first that $C(x)$ is finite when $x$ is a quadratic irrationality.

LEMMA 2. If $x$ belongs to a quadratic number field, then $0 \notin C(x)$ and $C_{S}(x)$ and $C(x)$ are finite.

This Lemma is essentially due to Lekkerkerker [5], see also Perron [6], p.6. The following proof contains an explicit representation of the elements of $C_{s}(x)$.

PROOF. $x=\left[b_{0}, b_{1}, \ldots\right]$ is represented in this case by a periodic continued fraction, i.e. $x=\left[b_{0}, \ldots, b_{r-1}, p_{0}, \overline{p_{1}, \ldots, p_{k-1}}\right], r \geq 1, k \geq 1$. It follows that $b_{r+n k+v}=p_{v}$ for $v=0,1, \ldots, k-1, n \in N_{0}$, and if $x_{v}=\left[\overline{p_{v}, p_{v+1}, \ldots, p_{k-1}, p_{o}, \cdots, p_{v-1}}\right]$, then $\xi_{r+n k+v}=x_{v}$ It follows from (4) that $\rho_{n}=\left[b_{n}, b_{n-1}, \ldots, b_{1}\right]$, hence $\rho_{r+n k+v-1} \rightarrow\left[\bar{p}_{v-1}, \mathrm{P}_{v-2}, \cdots, \mathrm{p}_{0}, \mathrm{p}_{\mathrm{k}-1}, \ldots, \mathrm{p}_{v}\right]=c_{v}(\mathrm{n} \rightarrow \infty)$, and the statement of Lemma 2 follows from (3).

REMARK. It follows from a theorem of Galois $([7], \S 23)$ that $c_{v}=-\frac{1}{\bar{x}_{v}}$, where $\bar{x}_{v}$ is the conjugate of $x_{v}$. Hence, the elements of $C_{s}$ are

$$
\frac{(-1)^{r+v-1}}{x_{v}-\bar{x}_{v}} \quad \text { if } k \text { is even, } \frac{ \pm 1}{x_{v}-\bar{x}_{v}} \quad \text { if } k \text { is odd. }
$$

This formula leads to an even more explicit representation of the elements of $C_{s}(x)$. 
This representation uses the notation $A_{n, j} / B_{n, j}$ for the convergents of $\left[b_{j}, b_{j+1}, \ldots\right]([7], \S 5)$. Let $A_{n} / B_{n}$ denote the convergents of $\left[\overline{p_{0}, \cdots, p_{k-1}}\right]$. Then the elements of $C_{s}(x)$ are

$$
\left\{\begin{array}{l}
(-1)^{r+v-1} \frac{B_{k-1, \nu}}{\sqrt{D}} \text { if } k \text { is even, } \pm \frac{B_{k-1, \nu}}{\sqrt{D}} \text { if } k \text { is odd, } \\
\nu=0,1, \ldots, k-1, D=\left(A_{k-1}+B_{k-2}\right)^{2}+4(-1)^{k-1}
\end{array}\right.
$$

In fact, we have $x_{v}=\frac{A_{k-1, \nu}-B_{k-2, \nu}+\sqrt{D_{\nu}}}{2 B_{k-1, \nu}}, D_{\nu}=\left(A_{k-1, \nu}+B_{k-2, \nu}\right)^{2}+4(-1)^{k-1}$ $([7], \oint 19)$. But $B_{i, j}=A_{i-1, j+1}, A_{i, j}=b_{j} A_{i-1, j+1}+B_{i-1, j+1} \quad([7], \S 5)$, and it follows that

$$
\begin{aligned}
& A_{k-1, v-1}+B_{k-2, v-1}=b_{v-1} A_{k-2, v}+B_{k-2, v}+A_{k-3, v}=b_{k-1+v} A_{k-2, v}+A_{k-3, v}+B_{k-2, v} \\
& =A_{k-1, v}+B_{k-2, v} \text {. Hence } D_{v}=D_{0} \text {, and (22) follows. }
\end{aligned}
$$

4. THE RELATION BETWEEN THREE CONSECUTIVE $\delta$ 's.

Formula (8) shows that $b_{n+1}$ is a function of $\delta_{n-1}, \delta_{n}, \delta_{n+1}$. The following Lemma shows that $b_{n+1}$ is also a function of $\delta_{n-1}, \delta_{n}$ alone. This fact is the key to the following considerations, which will show that the converse of Lemma 2 is also true.

LEMMA 3. For $\mathrm{n} \geq 0$

$$
b_{n+1}=\phi\left(\delta_{n-1}, 0 ; \delta_{n}\right) \quad \text {, and } \phi\left(\delta_{n-1}, 0 ; \delta_{n}\right) \notin N
$$

PROOF. Formulas (3), (6) and (8) imply

$$
\xi_{n+1}=\frac{1}{\delta_{n}}-\frac{1}{\rho_{n}}=\frac{1+\sqrt{1-4 \delta_{n} \delta_{n-1}}}{2 \delta_{n}}=\phi\left(\delta_{n-1}, 0 ; \delta_{n}\right) \quad(n \geq 0)
$$


and (23) follows from $\xi_{n+1}=\left[b_{n+1}, b_{n+2}, \ldots\right], \quad b_{n+1}=\left[\xi_{n+1}\right]$ (note that $\xi_{n+1}$ is irrational).

REMARK. Formulas (6) and (4) show that

$$
\phi\left(\delta_{n+1}, 0 ; \delta_{n}\right)=\frac{1+\sqrt{1-4 \delta_{n} \delta_{n+1}}}{2 \delta_{n}}=\rho_{n+1}=\left[b_{n+1}, b_{n}, \ldots, b_{1}\right] \quad(n \geq 0)
$$

and it follows

$$
b_{n+1}=\phi\left(\delta_{n+1}, 0 ; \delta_{n}\right), \phi\left(\delta_{n+1}, 0 ; \delta_{n}\right) \notin N,
$$

if $\mathrm{n} \geqslant 2$ or if $\mathrm{n}=1, \mathrm{~b}_{1}>1$.

The first formula (24) remains true for $n=0$.

Lemma 3 shows that a (universal) function $\Psi$ exists such that

$$
b_{n+1}=\psi\left(\delta_{n}, \delta_{n-1}\right), \quad n \geq 0,
$$

and the remark shows that also $b_{n+1}=\Psi\left(\delta_{n}, \delta_{n+1}\right)$ unless $n=1, b_{1}=1$, i.e. unless $\mathrm{n}=1, \delta_{0}>1 / 2$.

It follows from $(8)$ that $\psi\left(\delta_{n}, \delta_{n-1}\right)=\phi\left(\delta_{n-1}, \delta_{n+1}, \delta_{n}\right)$, hence there exists by (10) a function $X$ such that

$$
\delta_{n+1}=x\left(\delta_{n}, \delta_{n-1}\right), \quad n \geq 0,
$$

and similarly

$$
\delta_{n-1}=x\left(\delta_{n}, \delta_{n+1}\right) \text { unless } n=1, b_{1}=1
$$

Using the function $\Psi$, we find explicitely

$$
\delta_{n+1}=x\left(\delta_{n}, \delta_{n-1}\right)=\frac{1}{4 \delta_{n}}\left[1-\left(2 \delta_{n} \Psi\left(\delta_{n}, \delta_{n-1}\right)-\sqrt{1-4 \delta_{n-1} \delta_{n}}\right)^{2}\right]
$$

The following theorem gives $\Psi$ in a more convenient form than Lemma 3 . THEOREM 2. Let $n \geq 0, k_{n}=\left[\frac{1}{\delta_{n}}\right]$. Then $\delta_{n-1} \neq k_{n}\left(1-k_{n} \delta_{n}\right)$ and 


$$
b_{n+1}=\Psi\left(\delta_{n}, \delta_{n-1}\right)= \begin{cases}k_{n} & \text { if } \delta_{n-1} \in\left[0, k_{n}\left(1-k_{n} \delta_{n}\right)\right) \\ k_{n}-1 & \text { if } \delta_{n-1} \in\left(k_{n}\left(1-k_{n} \delta_{n}\right),\left(1-\delta_{n}\right)\right)\end{cases}
$$

PROOF. Assume that $\delta_{n-1}=k_{n}\left(1-k_{n} \delta_{n}\right)$. Then

$$
\phi\left(\delta_{n-1}, 0 ; \delta_{n}\right)=\frac{1+\sqrt{\left(2 \delta_{n n} k^{-1}\right)^{2}}}{2 \delta_{n}}=k_{n}
$$

(note that $2 \delta_{n} k_{n}>1$ ) which contradicts (23).

Let $\delta_{n-1} \in\left[0, k_{n}\left(1-k_{n} \delta_{n}\right)\right)$. Then by (10) and (28)

$$
k_{n}+1>\frac{1}{\delta_{n}}=\phi\left(0,0 ; \delta_{n}\right) \geq \phi\left(\delta_{n-1}, 0 ; \delta_{n}\right)>\phi\left(k_{n}\left(1-k_{n} \delta_{n}\right), 0 ; \delta_{n}\right)=k_{n} \text { and }
$$

$k_{n}=b_{n+1}$ follows from Lemma 3 .

Let $\delta_{n-1} \in\left(k_{n}\left(1-k_{n} \delta_{n}\right), 1-\delta_{n}\right)$ which 1mp1ies $n \geq 1$ since $\delta_{-1}=0$. Then, by (28), (10), (5) and (12)

$$
\begin{aligned}
k_{n}=\phi_{\phi}\left(k_{n}\left(1-k_{n} \delta_{n}\right), 0 ; \delta_{n}\right) & >\phi\left(\delta_{n-1}, 0 ; \delta_{n}\right) \geq \phi\left(1-\delta_{n}, 0 ; \delta_{n}\right) \\
& \geq \phi\left(0,0 ; \delta_{n}\right)-1=\frac{1}{\delta_{n}}-1>k_{n}-1,
\end{aligned}
$$

and $k_{n}-1=b_{n+1}$ follows from Lemma 3 .

Figure 1 shows the areas of constancy for the function $\Psi$.

\section{THE INFLUENCE OF $0 \notin C(x)$.}

Our next step is to introduce the assumption $0 \notin C(x)$, 1.e. $\delta_{n} \geq \lambda>0$, $\mathrm{n} \in \mathbf{N}$, for some $\lambda$ into our considerations.

LEMMA 4. Let $0 \leq \lambda \leq 1 / \sqrt{2}$.

If $n \geq 1$ and if $\delta_{n-1}$ and $\delta_{n+2}$ are $>\lambda$, then

4) This interval is empty if $k_{n}=1$. 


$$
\delta_{n}+\delta_{n+1}<\sqrt{1-\lambda^{2}}
$$

PROOF. Our proof depends on the inequality

$$
\phi\left(\lambda, \sqrt{1-\lambda^{2}}-z ; z\right) \leq 1 \text { if } \frac{1}{2} \sqrt{1-\lambda^{2}} \leq z<1,4 \lambda z<1,
$$

In order to prove (30) we observe that

$$
\begin{aligned}
& \sqrt{1-4 \lambda z \leq \sqrt{1-2 \lambda \sqrt{1-\lambda^{2}}}}=\sqrt{\left(\sqrt{1-\lambda^{2}}-\lambda\right)^{2}}=\sqrt{1-\lambda^{2}}-\lambda, \\
& \sqrt{1-4 z\left(\sqrt{1-\lambda^{2}}-z\right)}=\sqrt{\left(2 z-\sqrt{1-\lambda^{2}}\right)^{2}+\lambda^{2}} \leq\left(2 z-\sqrt{1-\lambda^{2}}\right)+\lambda,
\end{aligned}
$$

and (30) follows from

$$
\phi\left(\lambda, \sqrt{1-\lambda^{2}}-z ; z\right) \leq \frac{1}{2 z}\left(\sqrt{1-\lambda^{2}}-\lambda+2 z-\sqrt{1-\lambda^{2}}+\lambda\right)=1 .
$$

Assume that the assumptions of Lemma 4 hold and that $\delta_{n}+\delta_{n+1} \geq \sqrt{1-\lambda^{2}}$. If $\delta_{n} \geq \frac{1}{2} \sqrt{1-\lambda^{2}}$, then by (8), (10) and (30)

$$
b_{n+1}=\phi\left(\delta_{n-1}, \delta_{n+1} ; \delta_{n}\right)<\phi\left(\lambda, \delta_{n+1} ; \delta_{n}\right) \leq \phi\left(\lambda, \sqrt{1-\lambda^{2}}-\delta_{n} ; \delta_{n}\right) \leq 1,
$$

but $b_{n+1} \geq 1$.

Similarily, if $\delta_{n+1} \geq \frac{1}{2} \sqrt{1-\lambda^{2}}$,

$$
b_{n+2}=\phi\left(\delta_{n}, \delta_{n+2} ; \delta_{n+1}\right)<\phi\left(\lambda, \delta_{n} ; \delta_{n+1}\right) \leq \phi\left(\lambda, \sqrt{1-\lambda^{2}}-\delta_{n+1} ; \delta_{n+1}\right) \leq 1,
$$

but $\mathrm{b}_{\mathrm{n}+2} \geq 1$.

REMARK. Formula (5) is for $\mathrm{n} \geq 2$ a special case of (29). If $\delta_{n}>\lambda$ for all $n$, then it follows form (29) that $2 \lambda<\sqrt{1-\lambda^{2}}$, hence $\lambda<1 / \sqrt{5}$.

Lemma 4 will be used now to show that the points $\left(\delta_{n}, \delta_{n-1}\right)$ keep a certain distance from the discontinuities of $\Psi$ if $0 \notin C(x)$. We introduce the notation 


$$
\eta_{n}=k_{n}\left(1-k_{n} \delta_{n}\right) \text {, }
$$

and we assume chat $\delta_{\mathrm{n}}>\lambda>0$ for some $\lambda>0$ and all $\mathrm{n} \in \mathbf{N}$. Let $\delta_{n} \quad 1 / 2$ for some fixed $n \geq 2$. Formula (8) and Theorem 2 imply

$$
\sqrt{1-4 \delta_{n} \delta_{n-1}}+\sqrt{1-4 \delta_{n} \delta_{n+1}}=\left\{\begin{array}{lll}
2 \delta_{n} k_{n} & \text { if } & \delta_{n-1}<n_{n} \\
2 \delta_{n} k_{n}-2 \delta_{n} & \text { if } & \delta_{n-1}>n_{n}
\end{array}\right\}
$$

In what follows we need the inequality $2 \mathrm{n}_{\mathrm{n}}>\frac{2 \mathrm{k}_{\mathrm{n}}}{\mathrm{k}_{\mathrm{n}}+1} \geq \frac{4}{3}$ (note that $\mathrm{k}_{\mathrm{n}} \geq 2$ ) and the formulas $1-4 \delta_{n} n_{n}=\left(2 \delta_{n} k_{n}-1\right)^{2}, 1-4 \delta_{n}^{n}\left(1-\delta_{n}\right)=\left(1-2 \delta_{n}\right)^{2}$.

Let $\delta_{n-1}>\eta_{n}$, Then it follows from (31) that

$$
\sqrt{1}-\sqrt{1-4 \delta_{n} \delta_{n+1}}=\sqrt{1-4 \delta_{n} \delta_{n-1}}-\sqrt{1-4 \delta_{n} n_{n}} \text {, }
$$

hence (use $\sqrt{a}-\sqrt{b}=(a-b) /(\sqrt{a}+\sqrt{b})$ )

$$
\frac{\lambda}{2} \leq \frac{\delta_{n+1}}{2} \leq \frac{\delta_{n+1}}{1+\sqrt{1-4 \delta_{n} \delta_{n+1}}}=\frac{n_{n}-\delta_{n-1}}{\sqrt{1-4 \delta_{n} \delta_{n-1}}+\left(2 \delta_{n} k_{n}-1\right)} \leq \frac{n_{n}-\delta_{n-1}}{1 / 3} .
$$

It follows that

$$
\delta_{n-1} \leq n_{n}-\frac{\lambda}{6}
$$

Let $\delta_{n-1}>\eta_{n}$. Then it follows from (31) that

$$
\sqrt{1-4 \delta_{n} n_{n}}-\sqrt{1-4 \delta_{n} \delta_{n-1}}=\sqrt{1-4 \delta_{n} \delta_{n+1}}-\sqrt{1-4 \delta_{n}\left(1-\delta_{n}\right)} \text {, }
$$

hence, by Lemma 4

$$
\frac{\delta_{n-1}-n_{n}}{1 / 3} \geq \frac{\delta_{n-1}-n_{n}}{2 \delta_{n} k_{n}-1+\sqrt{1-4 \delta_{n} \delta_{n-1}}}=\frac{1-\left(\delta_{n}+\delta_{n+1}\right)}{1-4 \delta_{n} \delta_{n+1}+\left(1-2 \delta_{n}\right)} \geq \frac{1-\sqrt{1-\lambda^{2}}}{2} \text {. }
$$

It follows that 


$$
\delta_{n-1} \geq n_{n}+\frac{1-\sqrt{1-\lambda^{2}}}{6}
$$

Formula (4) implies that all points $\left(\delta_{n}, \delta_{n-1}\right), n \geq 2$, are in a certain open triangle, and some straight lines inside of this triangle are excluded by Theorem 2 (cf. figure 1).

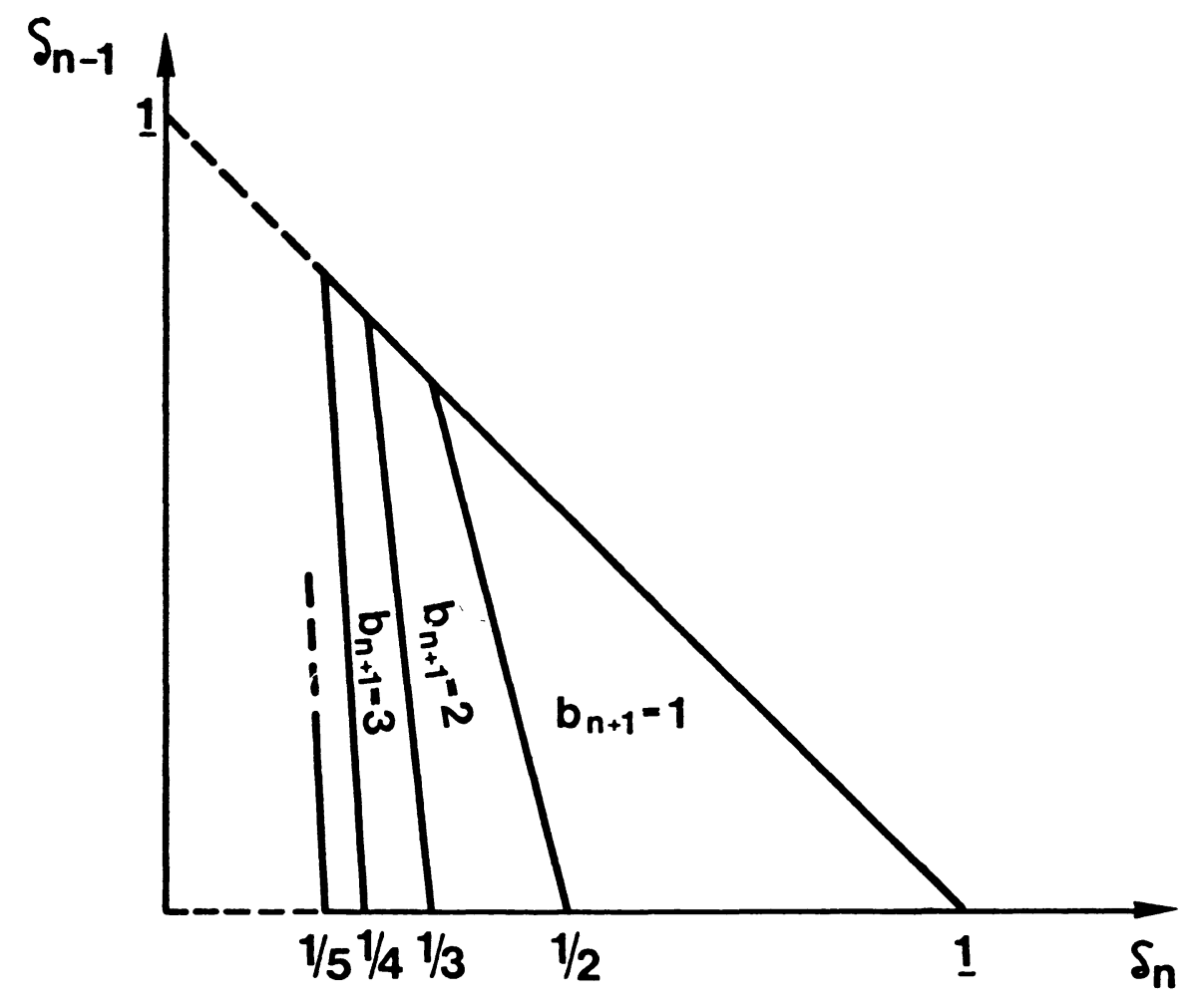

Fig. 1

Moreover, if $\delta_{\mathrm{n}}>\lambda>0$, then (29), (32) and (33) introduce some additional restriction for $\left(\delta_{n}, \delta_{n-1}\right)$. To describe the remaining region we introduce the following set.

Let $M(\lambda), 0 \leq \lambda<1 / \sqrt{5}$, denote the (open) set of points (x,y) with the properties 


$$
x>\lambda, y>\lambda, x+y<\sqrt{1-\lambda^{2}}
$$

and for $x<1 / 2$

$$
y<\left[\frac{1}{x}\right]\left(1-x\left[\frac{1}{x}\right]\right)-\frac{\lambda}{6} \quad \text { or } \quad y>\left[\frac{1}{x}\right]^{\cdot}\left(1-x\left[\frac{1}{x}\right]\right)+\frac{1-\sqrt{1-\lambda^{2}}}{6}
$$

(Figure 2 illustrates $M(\lambda)$ for $\lambda=1 / 5$. )

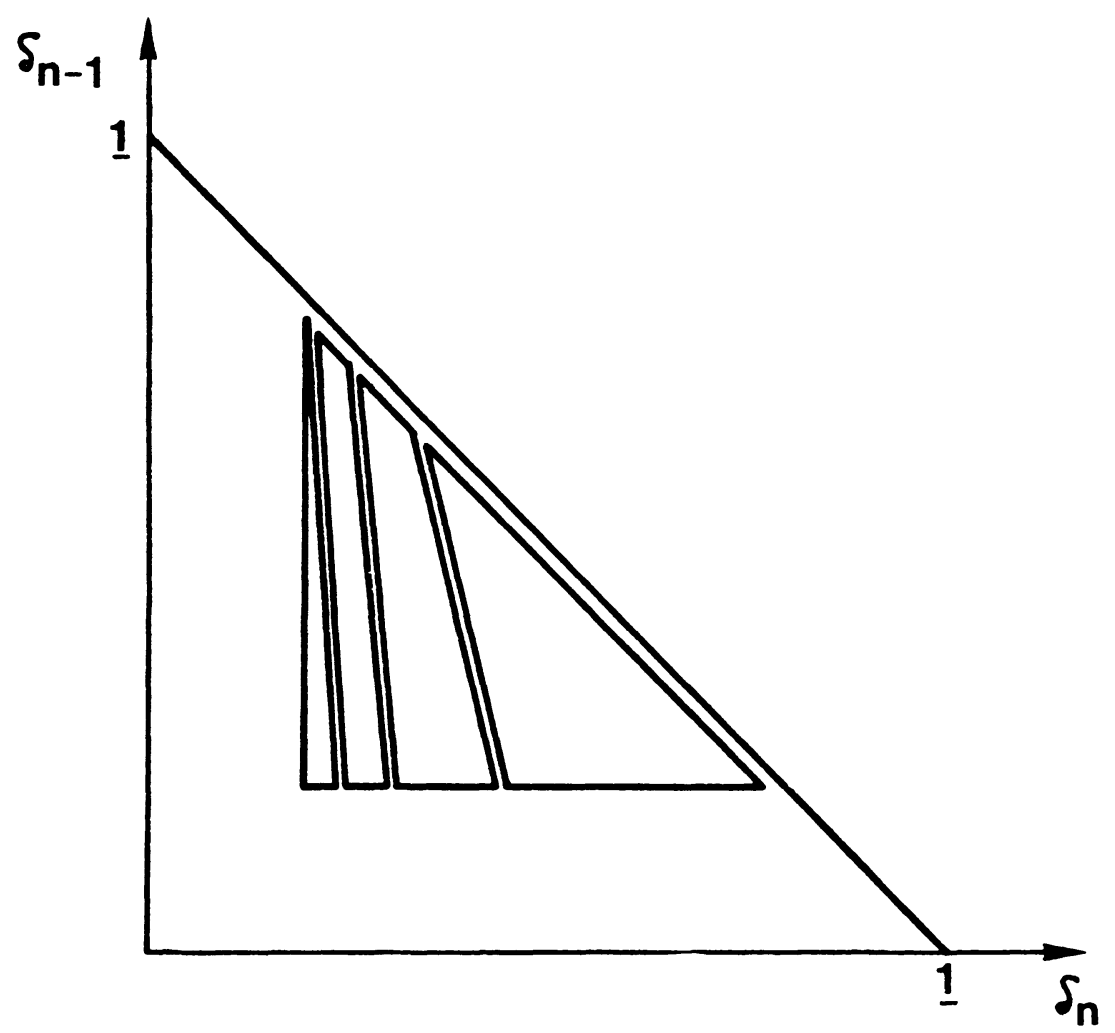

Fig. 2

If $\delta_{n}>\lambda \geq 0$ for all $n \in \mathbb{N}$, then $\left(\delta_{n}, \delta_{n-1}\right) \in M(\lambda)$ for $n \geq 3$ by (29), (32) and (33). The combination of this result with the results of section 4 leads immediately to

THEOREM 3. There are (universal) functions $\Psi$ and $X$, defined on $M(0)$, such that $b_{n+1}=\Psi\left(\delta_{n}, \delta_{n-1}\right), \delta_{n+1}=\chi\left(\delta_{n}, \delta_{n-1}\right), n \geq 0$. 
The functions $\Psi$ and $X$ are continuous on every $M(\lambda), \lambda>0$. If $\delta_{n}>\lambda>0(\lambda<1 / \sqrt{5})$ for all $n \in \mathbb{N}$, then $\left(\delta_{n}, \delta_{n-1}\right) \in M(\lambda)$ for $n \geq 3$.

\section{THE CONVERSE OF LEMMA 2.}

We use Theorem 3 to prove the following result of Lekkerkerker [5].

THEOREM 4. If $\mathrm{C}_{\mathrm{S}}(\mathrm{x})$ is finite and $0 \notin \mathrm{C}_{\mathrm{S}}(\mathrm{x})$, then $\mathrm{x}$ belongs to a quadratic number field.

PROOF. Let $\alpha_{i}$ denote the elements of $C(x)$, and let $A$ be the set to all pairs $\left(\alpha_{i}, \alpha_{j}\right)$ with $\left(\delta_{n}, \delta_{n-1}\right) \rightarrow\left(\alpha_{i}, \alpha_{j}\right)$ on a subsequence. Since $0 \notin c(s)$, there is some $\lambda>0$ such that $\left(\delta_{n}, \delta_{n-1}\right) \in M(\lambda)$ for all large $n$, and $a \in M(\lambda)$ for every $a \in A$. If $a=\left(\alpha_{i}, \alpha_{j}\right) \in A$ then $a^{\prime}=\left(x\left(\alpha_{i}, \alpha_{j}\right), \alpha_{i}\right) \in A$ since $\delta_{n_{k}} \rightarrow \alpha_{i}$, $\delta_{n_{k}-1} \rightarrow \alpha_{j}$ implies $\delta_{n_{k}+1}=x\left(\delta_{n_{k}}, \delta_{n_{k}-1}\right) \rightarrow\left(\alpha_{i}, \alpha_{j}\right)$ by Theorem 3 .

We call $a^{\prime}$ the successor of $a$. The set $A$ is finite, hence if $a \in A$ then one of its later successors is again a .

Let $\mathrm{U}(\mathrm{a}, \varepsilon)=\{(\mathrm{x}, \mathrm{y})||(\mathrm{x}, \mathrm{y})-\mathrm{a} \mid<\varepsilon\}, \mathrm{a} \in \mathrm{A}$. Choose $\varepsilon>0$ such that $u(a, \varepsilon) \subseteq M(\lambda)$ for every $a \in A, U(a, \varepsilon) \cap U(b, \varepsilon)=\emptyset$ if $a \neq b$.

It follows that $\Psi$ is constant on every $U(a, \varepsilon)$. Choose $\varepsilon^{*} \epsilon(0, \theta)$ such that for every $a \in A$

$$
\left\{(x(x, y), x) \mid(x, y) \in U\left(a, \varepsilon^{\star}\right)\right\} \subseteq U\left(a^{\prime}, \varepsilon\right) .
$$

Let $N \in \mathbb{N}$ be so large that $\left(\delta_{n}, \delta_{n-1}\right) \in U\left(a, \varepsilon^{*}\right)$ for exactly one a $\in A$ depending on $n \geq N$. This establishes a mapping $a=F\left(\delta_{n}, \delta_{n-1}\right)$ for every $\mathrm{n} \geq \mathrm{N}$ which is "successor preserving", i.e. if $\mathrm{F}\left(\delta_{\mathrm{n}}, \delta_{\mathrm{n}-1}\right)=\mathrm{a}$ then $F\left(\delta_{n+1}, \delta_{n}\right)=a^{\prime}$. Indeed, if $F\left(\delta_{n}, \delta_{n-1}\right)=a$, i.e. $\left(\delta_{n}, \delta_{n-1}\right) \in U\left(a, \varepsilon^{*}\right)$, then $\left(\delta_{n+1}, \delta_{n}\right)=\left(x\left(\delta_{n}, \delta_{n-1}\right), \delta_{n}\right) \leqq U\left(a^{\prime}, \varepsilon\right)$ by $(34)$, hence $\left(\delta_{n+1}, \delta_{n}\right) \in U\left(a^{\prime}, \varepsilon^{*}\right)$ since $\mathrm{n} \geq \mathrm{N}$. 
Take a fixed $n \geq N$, and let $a=F\left(\delta_{n}, \delta_{n-1}\right)$. Consider a sequence of successors $a=a^{(0)}, a^{\prime}, a^{\prime \prime}, \ldots, a^{(\ell)}, \ell \in \mathbf{N}$, with $a^{(\ell)}=a$. It follows that

$$
F\left(\delta_{n+\nu+k \ell}, \delta_{n-1+v+k \ell}\right)=a^{(\nu)} \quad, \quad \nu=0,1, \ldots, \ell-1, k=0,1,2, \ldots
$$

Since $\Psi$ is constant on every $U\left(a, \varepsilon^{*}\right)$, it follows from (35) that

$b_{n+v+k \ell+1}=\Psi\left(\delta_{n+v+k \ell}, \delta_{n+v+k \ell-1}\right)$ is independent of $k$, i.e. the continued fraction for $x$ is periodic. This proves Theorem 4 .

REMARK. As conclusion we explain our results in the simplest case $x=(1+\sqrt{5}) / 2=[1,1, \ldots]$. Here $c(x)$ consists of the single point $1 / \sqrt{5}$ by (22), and $D(x)$ consists of the points $\left|\lambda^{2}-\lambda \mu-\mu^{2}\right| / \sqrt{5}$ with integral $(\lambda, \mu) \neq(0,0)$ by $(16)$. It is well-known (see [3], p. 554) that

$$
\lambda^{2}-\lambda \mu-\mu^{2}=\left(\lambda-\mu \frac{1+\sqrt{5}}{2}\right)\left(\lambda-\mu \frac{1-\sqrt{5}}{2}\right)
$$

represents exactly the integers for which the exponents in the prime factorization must be even for all primes $\equiv 2$ or $3 \bmod 5$. So

$$
D(x)=\left\{\frac{1}{\sqrt{5}}, \frac{4}{\sqrt{5}}, \frac{5}{\sqrt{5}}, \frac{9}{\sqrt{5}}, \frac{11}{\sqrt{5}}, \frac{16}{\sqrt{5}}, \frac{19}{\sqrt{5}}, \frac{20}{\sqrt{5}}, \ldots\right\}
$$

Since this set contains only one element $\epsilon(0,1)$ it determines $C(x)$ uniquely. Furthermore, given $\mathrm{C}(\mathrm{y})=\{1 / \sqrt{5}\}$, all possible $\mathrm{y}$ which produce this set are given by integral transformations $y=\frac{a x+b}{c x+d}, \quad a d-b c= \pm 1$.

This follows because the proof of Theorem 4 works with $\ell=1$, so the continued fraction for $y$ has period 1 (the terms before the period being of no influence with quotients 1 by (22).

ACKNOWLEDGEMENT. This research was supported in part by the National Science Foundation. 


\section{REFERENCES}

1. Davis, N. and Kinney, J.R. : Quadratic Irrationals in the Lower Lagrange Spectrum. Can. J. Math. 25 (1973) 578-584.

2. Hardy, G.H. and Wright, E.M. : An Introduction to the Theory of Numbers. Oxford 1954 .

3. Hasse, H. : Zahlentheorie, Akademie-Verlag, Berlin 1969.

4. Jurkat, W.B., Kratz, W., and Peyerimhoff, A. : Explicit Representations of Dirichlet Approximations.

5. Lekkerkerker, C.G. : Una Questione di Approssimazione Diofantea e Una Proprieta Caratteristica dei Numeri Quadratici I, II.

Atti Accad. Naz. Lincei. Rend. Cl. Sci. Fis. Mat. Nat. (8) 21 (1956) 179-185, 257-262.

6. Perron, 0. : Uber die Approximation Irrationaler Zahlen Durch Rationale. S.-B. Heidelberger Akad. Wiss. Math.-Nat. K1. 12 (1921) 3-17.

7. Perron, 0. : Die Lehre von den Kettenbruchen. Teubner Verlag, Stuttgart $1954,1957$. 


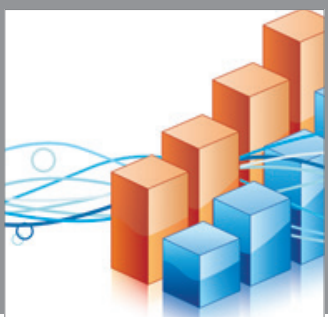

Advances in

Operations Research

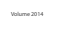

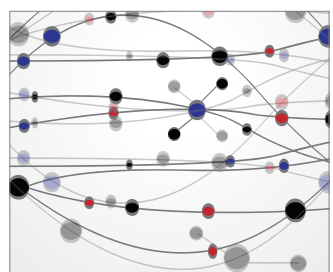

\section{The Scientific} World Journal
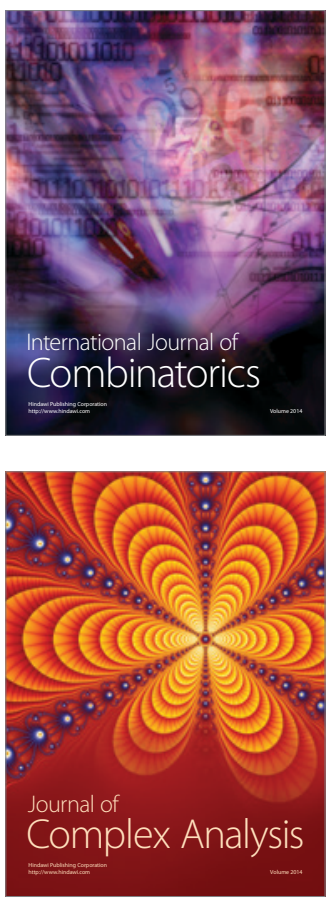

International Journal of

Mathematics and

Mathematical

Sciences
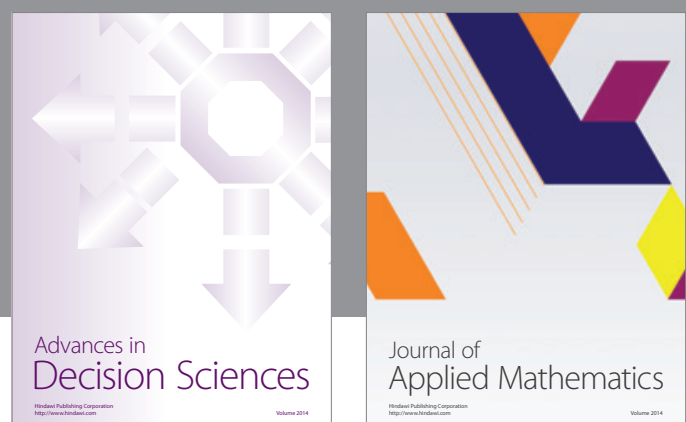

Journal of

Applied Mathematics
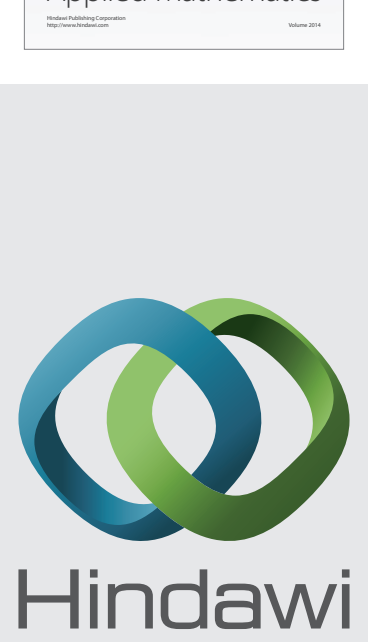

Submit your manuscripts at http://www.hindawi.com
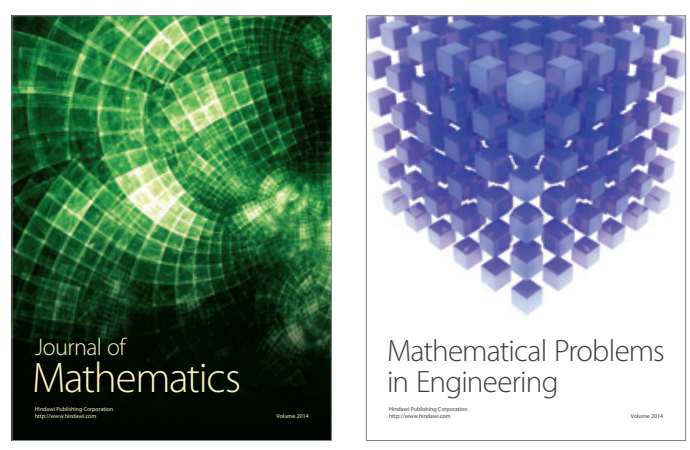

Mathematical Problems in Engineering
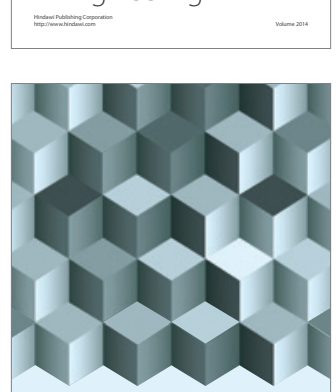

Journal of

Function Spaces
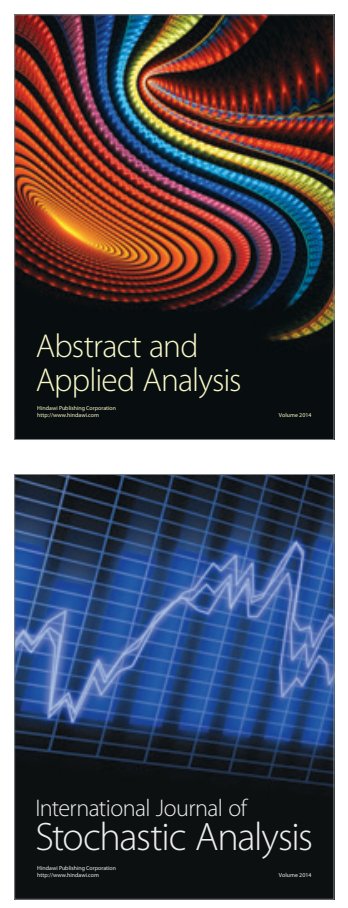

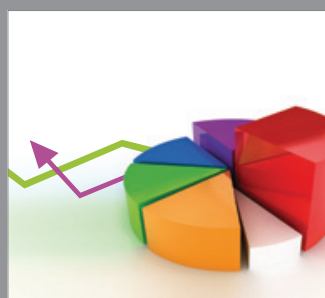

ournal of

Probability and Statistics

Promensencen
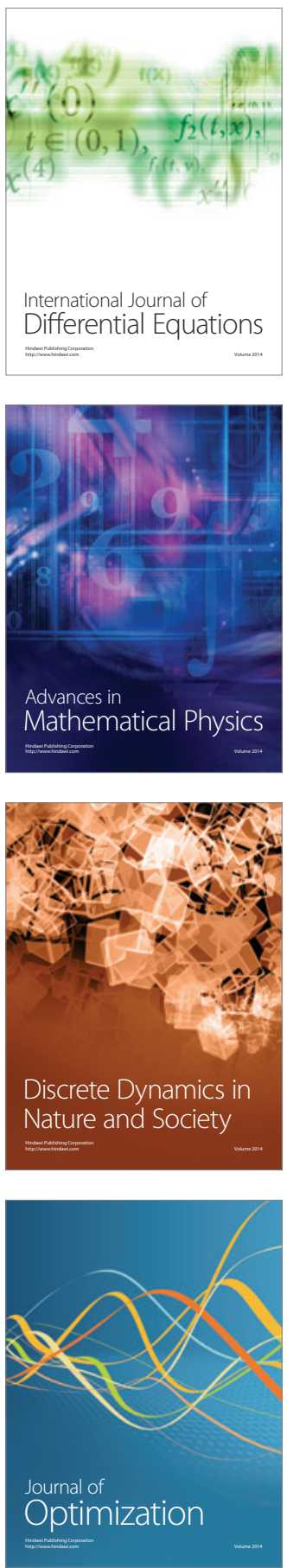